\title{
Connecting the hospital using 'Forward', a smartphone application for clinical teams
}

\author{
Authors: Joel Schamroth, ${ }^{\mathrm{A}}$ William Pettit, ${ }^{\mathrm{B}}$ Lydia Yarlott, ${ }^{\mathrm{C}}$ Lucinda Scharff, ${ }^{\mathrm{D}}$ Molly Gilmartin, ${ }^{\mathrm{E}}$ Rhydian Harris ${ }^{\mathrm{F}}$ and \\ Barnabas ] Gilbert ${ }^{\mathrm{E}}$
}

\begin{abstract}
Aims
A team of junior doctors aimed to create a smartphone messaging and workflow app, Forward, to improve the speed and security of communication between NHS clinical staff. Current options available to doctors include traditional systems (hospital landlines, radio-pagers) and unendorsed, non-compliant social messengers. To date, hospitals haven't capitalised on the widespread use of personal smartphones among clinical staff.
\end{abstract}

\section{Methods}

Research to prove the (i) problem / user case and (ii) effectiveness of the app (Forward) was undertaken over 1 year across multiple sites and using different research methods across three phases. A total of $>200$ clinical staff participated.

Phase 1: preliminary survey data (qualitative and quantitative), exploring (i) the use of smartphones in the clinical setting, (ii) existing communication bottlenecks/inefficiencies in hospitals and (iii) acceptability of a secure messaging solution.

Phase 2: qualitative and quantitative survey data following live simulation sessions on Forward (beta version).

Phase 3: multi-dimensional data (ethnographic, survey and push analytics) following live user testing within an acute NHS trust, exploring the effectiveness, safety and reliability of Forward (beta version).

Forward has been designed to handle data securely and to comply with NHS information governance (IG) requirements. All appropriate clinical safety and IG standards have been met and externally audited.

\section{Results}

From the initial survey data based on $>200$ doctor hours: $100 \%$ of doctors surveyed at work use their smartphone at work with $>95 \%$ using a non-compliant tool (eg WhatsApp) to augment clinical work. Doctors on average wasted 42 minutes/day

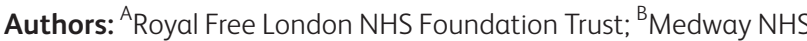
Foundation Trust; ${ }^{C}$ Homerton University Hospital NHS Foundation Trust; ${ }^{D}$ London North West University Healthcare NHS Trust; ${ }^{E}$ Oxford University Hospitals NHS Foundation Trust; ${ }^{{ }^{F}}$ Kingston Hospital NHS Foundation Trust, UK resulting from inefficient communication tools (radio-pagers, switchboards, paper lists). Smartphone messaging solutions were viewed as both acceptable and necessary by $>95 \%$ of clinical staff surveyed. Following live app testing, Forward is regarded as a viable messaging and workflow tool for clinical staff, in regards to speed and efficiency. Forward pilots in clinical settings (eg acute medicine departments) are live in several NHS trusts with preliminary data showing that the use of Forward can save each doctor >30 minutes time daily and significantly reduce use of noncompliant messaging alternatives.

\section{Conclusion}

In the fast-paced, high-pressure environment of modern NHS hospitals, clinical teams must care for an increasing number of patients with high turnover. Digital innovations to improve communication are required; our survey data demonstrates that doctors are keen for smartphone solutions. Forward is designed to provide secure, compliant messaging for clinical teams. Data from user simulations and preliminary trials in the clinical setting suggest that Forward has the potential to provide significant value for clinical teams by improving speed and efficiency of communications.

\section{Conflict of interest statement}

Forward began as a quality improvement project at Medway Maritime Hospital. In order to expand the scope of the project, Forward has been established as a private company. This is vital in order to raise funding to hire a professional technical team and for costs associated with testing Forward and scaling the app. All authors of this abstract are clinicians currently working for Forward. 